\title{
Atividade anti-helmíntica de plantas nativas do continente americano: uma revisão
}

\author{
SOUSA, R.G. '; FALCÃO, H.S.2; BARBOSA FILHO, J.M.2; MELO DINIZ, M.F.F.1,2; BATISTA, L.M.1,2,* \\ ${ }^{1}$ Centro de Ciências da Saúde, Departamento de Ciências Farmacêuticas, Programa de Educação Tutorial - \\ PET Farmácia, Universidade Federal da Paraíba (UFPB), Cx. Postal 5009, CEP. 58051-970, João Pessoa, PB, \\ Brasil. ${ }^{2}$ Centro de Ciências da Saúde, Departamento de Ciências Farmacêuticas, Laboratório de Tecnologia \\ Farmacêutica, Universidade Federal da Paraíba (UFPB), Cx. Postal 5009, CEP. 58051-970, João Pessoa, PB, \\ Brasil. *rafaelgserafim@gmail.com
}

\begin{abstract}
RESUMO: O presente trabalho faz uma revisão de plantas nativas do continente americano com atividade anti-helmíntica com a finalidade de subsidiar pesquisas e o desenvolvimento de novos medicamentos na área de produtos naturais. Na literatura, foram citadas 22 famílias de plantas, tais como: Moraceae, Asteraceae e Cucurbitaceae, e estudadas cerca de 35 espécies, como a Ficus sp, Cucurbita máxima, e Ruta graveolens, usadas por sua alegada atividade anti-helmíntica. Destas espécies, 26 foram ativas contra alguns helmintos: Strongyloides stercolaris, Ancylostomidae, Trichostrongylus sp, Capillaria sp, Ascaris lumbricoides, Taenia saginata, Haemonchus contortus, e Hymenolepsis diminuta. Foi verificado maior número de estudos com parasitas de animais, possivelmente devido ao desenvolvimento de resistência dos helmintos aos tratamentos convencionais disponíveis.
\end{abstract}

Palavras-chave: plantas; atividade anti-helmíntica; Continente americano; revisão.

ABSTRACT: Anthelmintic activity of native American plants: a review. This paper is a review of native American plants showing anthelmintic activity in order to give support to research and development of new drugs in the field of natural products. In the literature, 22 plant families were cited, such as: Moraceae, Asteraceae and Cucurbitaceae, and around 35 species were studied, including Ficus sp, Cucurbita maxima and Ruta graveolens, used for their putative anthelmintic activity. From these species, 26 were active against some helminthes: Strongyloides stercolaris, Ancylostomidae, Trichostrongylus sp, Capillaria sp, Ascaris lumbricoides, Taenia saginata, Haemonchus contortus and Hymenolepsis diminuta. Data indicated a larger number of studies with animal parasites, probably due to the resistance of these helminthes to the conventional treatments available.

Key-words: plants, anthelmintic activity, America, review.

\section{INTRODUÇÃO}

As propriedades terapêuticas de produtos naturais derivados de plantas, minerais e animais foram evidenciadas desde as mais antigas civilizações humanas. Atualmente, as plantas medicinais são as principais fontes naturais para a síntese de medicamentos, o que lhes confere destaque no panorama americano e mundial (Reis et al., 2004).

Segundo a Organização Mundial da Saúde (2002), as plantas medicinais são utilizadas por $80 \%$ da população mundial na atenção primária à saúde. Nas últimas décadas, a produção de fitoterápicos tem aumentado nas indústrias de países desenvolvidos. Os países europeus, especialmente a Alemanha, asiáticos e os Estados Unidos, possuem os principais mercados produtores e consumidores desses medicamentos (TORRES; SOUZA, 2010).

Países em desenvolvimento como o Brasil não apresentam uma produção industrial marcante de medicamentos ou fitoterápicos, no entanto, a instituição da Política Nacional de Plantas Medicinais e Fitoterápicos, PNPMF, (Decreto $n^{\circ}$ 5.813, 22 de junho de 2006) tem como medida fortalecer o desenvolvimento industrial e tecnológico nesta área e sobretudo garantir melhor acesso aos medicamentos pelos usuários do SUS através de programas básicos de saúde de forma a valorizar

Recebido para publicação em 07/04/2011

Aceito para publicação em 28/05/2012

Rev. Bras. PI. Med., Campinas, v.15, n.2, p.287-292, 2013. 
o conhecimento popular e incentivar essa terapia alternativa aos quimioterápicos (Ministério da Saúde, 2006).

Através do PNPMF, em 2009, foi publicada uma lista contendo 71 plantas medicinais que apresentam potencial para gerar produtos de interesse ao SUS (Relação Nacional de Plantas Medicinais de Interesse ao SUS-RENISUS). Dados científicos de algumas destas espécies torna possível sua validação tanto do ponto de vista químico quanto farmacológico o que está na base para o desenvolvimento de fitoterápicos (ZUANAZZI; MAYORGA, 2010).

Nos dias atuais, muitas plantas são utilizadas na sua forma bruta, fitoterápica ou como princípio ativo isolado. A espécie Eucalyptus globulus (Myrtaceae) tem ganhado destaque mundial na saúde e principalmente nos países em desenvolvimento por ser uma planta de tradição popular que ganha respaldo científico no combate a doenças do aparelho respiratório bem como apresenta potencial anti-helmíntica (SCHULZ, 2002). Além dessa, a Ficus glabrata H.B.K. (Moraceae) (figueira), que já tinha sido estudada em 1943 por Werner, apresentou eficácia potencial no controle da ascaridíase.

Já é constatado que as infecções por parasitas intestinais são reflexos das condições de vida das diferentes comunidades (Chieffi et al., 2003) e que constituem importantes entidades mórbidas para o homem e animais, pois têm ampla distribuição geográfica e, em alguns casos, elevados índices de prevalência (Botero, 1979; 1981). Esse fato é discutido há várias décadas. Por exemplo, um estudo elaborado por Melo e colaboradores (1988) na América Latina, evidenciou que o índice de doenças relacionadas às infecções parasitárias não se modificava durante anos e tinha como causa a distribuição geográfica dos parasitas concomitante ao subdesenvolvimento dos países, ou seja, é um indicativo das condições socioeconômicas das comunidades. Aguilar (1981) apresentou dados da Divisão de Epidemiologia dos Serviços de Saúde da Guatemala e verificou que as helmintíases intestinais encontravam-se entre as 10 principais causas de morbidade e mortalidade geral do país. No Chile, apesar de melhoria das condições de saneamento, a prevalência dos parasitas intestinais em algumas regiões de importância econômica para o país é bastante alta (Schenome et al., 1981).

Vinha (1975) ressaltou que a importância das parasitoses intestinais no Brasil não comporta contestação. Os índices são altos, há uma ampla distribuição geográfica da doença atingindo principalmente grupos da população de baixo padrão socioeconômico (Melo et al.,1988). Ainda como conseqüência dos padrões socioeconômicos, esses índices aumentam na população devido ao uso limitado dos medicamentos, uma vez que não são acessíveis às comunidades residentes em áreas rurais, distantes de centros comerciais ou postos públicos de distribuição de medicamentos, ou ainda os parasitos podem apresentar resistência aos medicamentos anti-helmínticos (CamurçaVasconcelos, 2005). Diante deste contexto, pesquisar as propriedades terapêuticas de plantas contra infecções parasitárias no homem e animais pode oferecer uma alternativa para minimizar alguns destes problemas (Maciel et al, 2006). Portanto, o presente artigo faz uma revisão literária de plantas nativas do continente americano com alegadas propriedades farmacológicas contra parasitoses intestinais em seres humanos e animais.

\section{FONTES DE DADOS}

Neste artigo, alguns relatórios sobre atividade anti-helmíntica de plantas estudadas no Continente Americano foram revistos em literatura especializada publicados até abril de 2012. A pesquisa foi realizada em bancos de dados como o Biological Abstracts, SciFinder Scholar, Periódicos da CAPES, Pubmed e Banco de Dados NAPRALERT (Acrônimo de Produtos Naturais ALERT - Universidade de Illinois, Chicago, E.U.A.). As referências foram consultadas para maiores detalhes dos modelos experimentais utilizados para testar os extratos, as doses, as atividades e organismo testado.

\section{RESULTADOS E DISCUSSÃO}

Nesta revisão podemos verificar que 35 espécies de plantas foram estudadas quanto à atividade anti-helmíntica, sendo 26 destas ativas para helmintos como Strongyloides stercolaris, Ancylostomidae, Trichostrongylus $\mathrm{sp}$, Capillaria sp, Ascaris lumbricoides, Taenia saginata, Haemonchus contortus e Hymenolepis diminuta. Também verificamos que as espécies botânicas estão distribuídas em 22 famílias: Acanthaceae, Amaranthaceae, Araucariaceae, Asteraceae, Bromeliaceae, Chenopodiaceae, Cucurbitaceae, Dryopteriadaceae, Ebenaceae, Fabaceae, Lamiaceae, Liliaceae, Loganiaceae, Meliaceae, Moraceae, Moringaceae, Malvaceae, Pentaphylacaceae, Rosaceae, Rutaceae, Solanaceae e Zygophillaceae (ver Quadro 1).

Um estudo elaborado por De Amorim e colaboradores (1999) mostrou que em camundongos tratados com látex de Ficus insípida Wild. (Moraceae), os percentuais de eliminação de Syphacia obvelata $(38,6 \%)$ e Aspiculuris tetraptera $(8,4 \%)$ não foram tão pronunciados, quando comparados aos controles. $O$

Rev. Bras. PI. Med., Campinas, v.15, n.2, p.287-292, 2013. 
látex de Ficus carica Linn. (Moraceae) exibiu uma atividade semelhante ao de F. insipida quanto à eliminação de S. obvelata (41,7\%). Entretanto, os helmintos $A$. tetraptera $(2,6 \%)$ e Vampirolepis nana $(8,3 \%)$ não foram afetados pelo látex de $F$. carica. O tratamento padrão utilizado neste estudo foi nitroscanato que apresentou taxa de eliminação de $96,7 \%$ para $A$. tetraptera, $100 \%$ para S. obvelata e $V$. nana. A atividade anti-helmíntica de $F$. insipida e F. carica contra S. obvelata deve-se provavelmente à ação proteolítica do látex, que digere parcialmente os oxiurídeos. No entanto, a utilização desses produtos na medicina tradicional para o tratamento de parasitoses intestinais não é recomendada, uma vez que nos animais tratados com látex de $F$. insipida ou $F$. carica surgiram microfocos hemorrágicos na mucosa do trato gastrintestinal (estômago, intestino delgado, ceco e cólon), indicativos de ação próulcerogênica, provavelmente, e a administração de concentrações de $10 \mathrm{ml} / \mathrm{kg}$ durante três dias causou letalidade de $60 \%$ dos animais.

Os resultados do trabalho de Cruz et al. (2008) mostram que o percentual de eliminação de A. tetraptera em camundongos após a administração de $0,04 \mathrm{~mL} / \mathrm{g}$ da infusão das folhas secas de Solanum lycocarpum L. (Solanaceae) por via intragástrica foi de $5.64 \pm 3.33 \%$. O controle negativo mostrou resultado de $1.56 \pm 3.16 \%$ e as drogas controle foram o nitroscanato e o mebendazol, com percentuais de eliminação de $64 \%$ e $100 \%$, respectivamente. É muito possível que os glicoalcalóides e agliconas, presentes nos extratos, uma vez ingeridos possam afetar o sistema reprodutivo dos helmintos. A atividade hipocolesterolêmica que a planta apresenta poderia ser devido a aumentada excreção ácida da bile fecal, bem como à ação de ácidos graxos de cadeia curta, produzidos por fermentação da síntese de alguns compostos como o delta-aminolevulinato e do colesterol 7-alfa-hidroxilase. Devido aos efeitos relatados, é bem provável que esses ácidos graxos seriam também responsáveis pelo efeito antihelmíntico.

Werner (1943) afirma que a seiva de Ficus species (Moraceae) não apresenta atividade antihelmíntica contra Ascaris, Necator, Trichocephalus, e Oxyuris de origem humana e Ancylostomum, em cães, quando testado em modelos in vitro. $\mathrm{O}$ autor relata que a ausência do componente ativo ficcina pode ser a razão da ineficácia das espécies estudadas.

Em estudo clínico, Hansson e colaboradores (1986) demonstraram que o látex de F. glabrata apresentou efeito letal sobre $A$. lumbricoides em concentrações abaixo de $0,05 \%$ em solução salina fisiológica. Esse ensaio incluiu 181 pessoas e resultou em uma dose recomendada de $1,0 \mathrm{ml}$ de solução salina de látex/ Kg de peso de preparado por dia, durante 3 dias para ser repetida a cada 3 meses. Em outro estudo clínico, Phillips (1990) utilizando o látex fresco da espécie $F$. insipida relatou que esta espécie não possui atividade anti-helmíntica contra A. lumbricoides. Ambos os estudos foram realizados em seres humanos (testes clínicos) e este fato proporciona maior respaldo à pesquisa $e$ índice de confiabilidade nos resultados, visto que em experimentos in vitro e também em estudos pré-clínicos em animais existem restrições.

A eficácia das sementes secas de Cucurbita maxima Duchesne ssp., Cucurbita moschata Duchesne ssp. e Cucurbita pepo Linn. (Cucurbitaceae) foi investigada contra Vampirolepsis species. A dose $20 \mathrm{mg} / \mathrm{kg}$, de cada espécie de planta, foi administrada por via oral e intragástrica a camundongos infectados. Nesse estudo, verificouse que nenhuma das plantas testadas apresentou atividade contra a V. species (De Amorim; Borba, 1992).

Em outro estudo, os extratos etéreos das folhas de Araucaria excelsa Lamb. (Araucariaceae) e do talo de Vanillosmopsis erythropappa Schult. Bip (Asteraceae) foram testados e ativos in vitro contra Strongyloides stercolaris. Os extratos etéreos das cascas, folhas e frutas de Trichilia lagoensis C.DC. (Meliaceae), das flores da Guarea sepium Linn. (Meliaceae), das folhas de Mentha spicata Linn. (Lamiaceae) e Ruta graveolens Linn. (Rutaceae) também foram testados e ativos in vitro contra este parasito (Gilbert et al., 1972).Visto que, não foi possível encontrar estudos in vivo em seres humanos da atividade anti-helmíntica de produtos naturais contra $S$. stercolaris, não há como se confrontar os dados ou mesmo apresentar uma análise da atividade anti-helmíntica mais fidedigna da infecção no homem.

Vichnewski e colaboradores (1982), também em modelos in vitro, conseguiram a inibição do desenvolvimento larvar de $S$. stercoralis e de três espécies de Ancylostomidae, utilizando o extrato acetato de etila obtido das partes aéreas de Calea lantanoides Gard. (Asteraceae).

Foi identificado por Kato (2000) que o óleo essencial da Chenopodium ambrosioides Linn. (Chenopodiaceae) administrado na concentração de $0,1 \mathrm{~mL} / \mathrm{kg}$ para ratos infectados com Strongyloides sp e Nematodirus sp não apresentou atividade. No entanto, este óleo na mesma concentração foi eficaz contra Trichostrongylus sp. Além da atividade anti-helmíntica nesta e em outras espécies, C. ambrosioides mostrou atividade molusquicida pronunciada contra o hospedeiro natural de Schistosoma mansoni, o caracol da espécie Biomphalaria glabrata (SANTOS et al.2012).

Em um estudo comparativo com Allium 
sativum Linn.(Liliaceae) em carpas, Peña e colaboradores (1988) evidenciaram que $200 \mathrm{mg}$ deste produto apresentou $100 \%$ de atividade antihelmíntica contra Capillaria sp. Já o extrato hexânico mostrou ser eficaz em $75 \%$ e o extrato aquoso não foi ativo. Esta diferença de atividade pode ser relacionada ao grau de polaridade dos componentes ativos de $A$. sativum, principalmente a aliina, que são melhores extraídos pelo hexano do que pela água.

Segundo Cáceres e colaboradores (1990), o extrato aquoso de qualquer parte da planta Moringa pterygosperma Gaertn.(Moringaceae) não apresenta atividade contra $A$. lumbricoides. No entanto, quando foi utilizado o pó das cascas secas dessa espécie foi verificada atividade contra este mesmo parasito em um estudo in vitro (Quadro 1).

Um estudo conduzido em 1943 por Hernandez e colaboradores relatou que o suco fresco das partes verdes de Ananas comosus (L.) Merr. (Bromeliaceae) apresentou atividade contra A. lumbricoides em modelo in vivo e também contra a Taenia saginata em seres humanos. Em uma pesquisa desenvolvida por Kliks (1985), com os extratos aquosos das folhas, florações e sementes secas de $C$. ambrosioides, foi verificado que estes produtos não apresentaram atividade contra $A$. lumbricoides. Bliss (1925), porém, considerou eficaz o óleo essencial desta espécie vegetal ( $1 \mathrm{~mL} / a n i m a l)$ contra este parasita em cães. É apresentada uma série de estudos sobre $A$. lumbricoides e de alguns não foi possível obter dados completos quanto aos modelos de ensaio, mesmo assim, relata-se a atividade das plantas avaliadas (ver Quadro 1).

Dados recentes indicam que o $A$. lumbricoides é o helminto mais frequente nos países pobres, sendo sua estimativa de aproximadamente $30 \%$, ou seja, 1,5 bilhão de pessoas em todo o mundo, atingindo cerca de 70 a $90 \%$ das crianças na faixa etária de 1 a 10 anos. Cerca de $8 \%$ das infecções por ascaris no mundo concentram-se na América latina. As condições climáticas tem bastante influência nas taxas de infecção. Em geral, a prevalência é baixa em regiões áridas, sendo, contudo relativamente alta onde o clima é úmido e quente, condição ideal para a sobrevivência e embrionamento dos ovos. Além disso, áreas desprovidas de saneamento com alta densidade populacional contribuem significativamente para o aumento da carga da doença (NEVES, 2010).

Ainda, Pessoa e colaboradores (2002) avaliaram as propriedades anti-helmínticas do óleo essencial de Ocimum gratissimum Linn. (Lamiaceae) em diferentes concentrações contra Haemonchus contortus em pequenos ruminantes (caprinos e ovinos). A porcentagem média de inibição na eclosão dos ovos para este parasita foi bastante significativa: Nas concentrações de
0.50 e 1,0\%, por exemplo, obteve-se eficácia de $100.0 \pm 6.13 \%$ e $100.0 \pm 9.22 \%$, respectivamente, semelhante ao controle positivo, tiabendazol $(0,5 \%)$, cujo resultado foi $100.0 \pm 0.0 \%$. Em outro trabalho também utilizando caprinos infectados com $\mathrm{H}$. contortus, o extrato etanólico das sementes de Melia azedarach Linn. (Meliaceae) foi o mais ativo $\left(\mathrm{CL}_{50}=0,36 \mathrm{mg} / \mathrm{mL}\right)$ contra os ovos deste parasita, enquanto o extrato etanólico da folha desta mesma planta apresentou as melhores taxas de inibição de desenvolvimento larvar $\left(\mathrm{CL}_{50}=9,18 \mathrm{mg} / \mathrm{mL}\right)$ (Maciel et al., 2006). Estudando o potencial da Spigelia anthelmia Linn. (Loganiaceae) contra $H$. contortus, Assis e colaboradores (2003) avaliaram os extratos desta planta nas concentrações 3.1, $6.2,12.5,25$ e $50 \mathrm{mg} / \mathrm{mL}$. Foi verificado que o extrato acetato de etila inibiu $100 \%$ dos ovos de incubação e $81.2 \%$ do desenvolvimento larvar desse parasita na concentração de $50 \mathrm{mg} / \mathrm{mL}$. De forma semelhante, o extrato metanólico inibiu $97.4 \%$ dos ovos de incubação e $84.4 \%$ das larvas em desenvolvimento. Estes resultados sugeriram que a utilização de $S$. anthelmia pode ser útil no controle de nematódeos gastrintestinais de ovinos e caprinos. Já no experimento conduzido por Worku, Franco e Miller (2009) o extrato aquoso das folhas de tabaco (Nicotiana tabacum L., Solanaceae, 200 $\mathrm{mg} / \mathrm{Kg}$,) adicionado a sulfato de cobre, foi ineficaz no combate ao $H$. contortus e Trichostrongylus spp. em caprino, além de ter sido demonstrada sua toxicidade ao diminuir o volume globular médio das células vermelhas do sangue.

Rios de Álvarez e colaboradores (2011) mostrou o efeito dos extratos aquosos das plantas tropicais secas Trichanthera gigantean Kunth (Acanthaceae), Gliricidia sepium jacq (Fabaceae) e Leucaena leucocephala (Lam.) de Wit (Fabaceae) sobre o parasitismo das larvas L1 de $H$. contortus. O G. sepium e L. leucocephala apresentaram um efeito dose-dependente com IC ${ }_{50}$ estimada de 0,015 $\mathrm{mg} / \mathrm{ml} \pm 0,001$ e $3,465 \mathrm{mg} / \mathrm{ml} \pm 0,144$. As lecitinas, componentes ativos, destas planta, podem ser as responsáveis pela propriedade inibitória do desenvolvimento de $L_{1}$ in vitro.

No trabalho com Dryopteris filix-mas (L.) Schott (Dryopteriadaceae) ficou evidenciada que a resina extraída do óleo desta espécie vegetal pode ser ativa contra Hymenolepis diminuta, quando administrada oralmente numa dose de $3 \mathrm{mg} / \mathrm{kg}$ em camundongos parasitados (Kim, 1972). Luttermoser e Bond (1957) avaliaram o potencial de três espécies da família Ebenaceae. Utilizando a polpa da fruta de Diospyros ebenaster Retz e D. virginiana Linn. em cães, descobriu-se que a $D$. ebenaster não apresentou qualquer atividade na dose de $0,5 \mathrm{mg} /$ $\mathrm{kg}$ por via oral contra Dipylidum caninum e Trichuris vulpis, assim como a $D$. virginiana na dose de

Rev. Bras. PI. Med., Campinas, v.15, n.2, p.287-292, 2013. 
$1 \mathrm{mg} / \mathrm{kg}$, pela mesma via, contra Toxocara canis e Ancylostoma caninum. As cascas da fruta de $D$. kaki e $D$. virginiana também foram inativas para $T$. canis e A. caninum.

Um estudo in vitro demonstrou que extratos, nas doses de 500 e $50 \mathrm{mg} / \mathrm{mL}$, das plantas Tilia mexicana L. (Malvaceae) obtiveram eficácia de 100; Crataegus mexicana DC (Rosaceae), 95 e 90\%; e Ternstroemia pringlei (Rose) Standl. (Pentaphylacaceae), 100\%, contra metacercárias encistadas de Fasciola hepatica. (VERAMONTENEGRO, et al., 2008)

E avaliando extrato metanólico da planta inteira seca de Amaranthus caudatus L. (Amaranthaceae), usando minhocas (Pheretima posthuma), foi observado que, em várias concentrações (10-100 mg/ml), o extrato demonstrou significativa atividade contra Pheretima posthuma, quando comparado à droga padrão piperazina (10 $\mathrm{mg} / \mathrm{ml}$ ). Os experimentos mostraram que o extrato foi capaz de causar diminuição da motilidade, paralisia e, finalmente, morte. A eficácia de $A$. caudatus deve-se muito provavelmente à presença de compostos polifenólicos. A atividade letal do extrato metanólico contra Pheretima sugere que o mesmo é efetivo contra helmintos em humanos, devido às similaridades entre a espécie Pheretima posthuma e anelídeos parasitas (SRINIVASA et al., 2010).

\section{CONCLUSÃO}

De todas as plantas estudadas, verificamos que a atividade anti-helmíntica foi comprovada por meio de estudos in vitro ou in vivo e que, dependendo da espécie da planta ou do parasita investigado, esta atividade foi ou não confirmada. Assim, ao se avaliar a atividade anti-helmíntica de extratos de plantas devem ser considerados no mínimo importantes fatores, como: tipo de extrato, parte da planta utilizada, concentração/dose, via de administração, bioensaio utilizado, espécie animal infectada e qual a espécie do parasita.

Verificamos que existe um maior número de estudos com helmintos cujos agentes hospedeiros são animais de experimentação (cobaias), murinos, caninos, felinos, aves, caprinos, peixes e bovinos, e poucas pesquisas relacionadas às verminoses humanas. Isso pode ser devido ao fato de que há uma maior resistência aos anti-helmínticos utilizados nos animais do que no ser humano (COELHO, 2009).

Sendo assim, a ineficácia do tratamento das verminoses naqueles acarreta muitos prejuízos à produção alimentícia, por isso haja, talvez, maior interesse em pesquisar princípios ativos mais eficientes (SANGSTER, 2001).

\section{AGRADECIMENTOS}

Os autores agradecem ao MEC-SESu pelo investimento ao Programa de Educação Tutorial (PET-Farmácia) e a Universidade de Illinois, Chicago - E.U.A., pela assistência com o banco de dados NAPRALERT.

\section{REFERÊNCIA}

AGUILAR, F.J. Helmintiasis humanas transmitidas através del suelo en Guatemala. Boletim Chileno del Parasitologia. Vol. 36. Pg. 6-9. 1981.

ASSIS, L.M.; BEVILAQUA, C.M.L.; MORAIS, S.M.; VIEIRA, L.S.; COSTA, C.T.C.; SOUZA, J.A. L. Ovicidal and larvicidal activity in vitro of Spigelia anthelmia Linn. extracts on Haemonchus contortus. Veterinary Parasitology. Vol. 117. Pg. 43-49. 2003.

BLISS JR, A.R. A pharmacodynamic study of the anthelmintic properties of western oils of chenopodium. Journal American Veterinary Medical. USA. Vol. 66. Pg. 625. 1925.

BOTERO, B. Posibilidades de control de las geohelmintíases mediante tratamientos en masa. Boletim Chileno del Parasitologia. Vol. 34. Pg. 3943. 1979.

BOTERO, D. Persistência de parasitoses intestinais endêmicas na América Latina. Boletim Oficinal Sanitário Panamericano. Vol. 60. Pg. 39-47. 1981.

CACERES, A; CABRERA, O; MORALES, O; MOLLINEDO, $P$; MENDIA, P. Pharmacological properties on moringa oleifera. 1: preliminary screening for antimicrobial activity. Journal of Ethnopharmacology. Guatemala. Vol. 33 (3) Pg. 213-216. 1990.

CAMURÇA-VASCONCELOS, A.L.F.; MORAIS, S.M.; SANTOS, L.F.L.; ROCHA, M.F.G.; BEVILAQUA, C.M.L. Validação de plantas medicinais com atividade antihelmíntica. Revista Brasileira de Plantas Medicinais. Brasil. Vol. 7. n. 3. Pg .97-106. 2005.

CHIEFFI, P.P.; NETO, V.A. Vermes, verminoses e a saúde pública. Ciências Culturais. Vol. 552003.

COELHO, W.A.C. Resistência anti-helmíntica em caprinos no município de Mossoró/RN. 2009. 57 pag. Dissertação (Mestrado em Ciência Animal: Área de concentração em Parasitologia) - Universidade Federal Rural do Semi-Árido. Mossoró. Disponível em: http:// www2.ufersa.edu.br/portal/view/uploads/setores/80/ Disserta\%C3\%A7\%C3\%A3o_Wesley_Adson_Costa_ Coelho.pdf. Acesso: 16 de maio de 2012.

CRUZ, A. P. S. et al. Anthelmintic effect of Solanum lycocarpum in mice infected with Aspiculuris tetraptera. The Journal of American Science. 2008. http://www. americanscience.org. Acesso em: 10 de abril de 2012.

DE AMORIM, A.; BORBA, H.R.; CARAUTA, J.P.P; LOPES, D; KAPLAN, M.A.C. Antihelmintic activity of the latex of Ficus species. Elsevier. Brazil. 1999.

DE AMORIM, A; BORBA, HR. Antihelmintic action of plants. Vi. Influence of pumpkin seeds in the removal of vampirolepis nana from mice. Revista Brasileira de Farmacognosia. 73(4). Pg. 81-82. 1992.

FERREIRA, S. H. Medicamentos à base de plantas medicinais. Academia Brasileira de Ciências. 2002. Disponível em http: www.abc.org.br/arquivos/

Rev. Bras. Pl. Med., Campinas, v.15, n.2, p.287-292, 2013. 
medicamentos.pdf Acesso: 20 fev 2010.

GALLOTTE, D. C.; RIBEIRO, L. F. Levantamento etnobotânico das plantas medicinais do horto da Escola Superior São Francisco de Assis - ESFA. Santa Teresa. 3(1): 19-24. Disponível em http://www.naturezaonline. com.br Acesso: 02 Mar 2010.

GILBERT, B; MORS, WB; BAKER, PM; TOMASSINI, TCB; COULART, EG; DE HOLANDA, JC; RIBEIRO DA COSTA, JÁ; LOPES, JNG; DOS SANTOS FILHO, D. Antihelmintic activity of essential oils and their chemical components. Academia Brasileira de Ciências. Vol. 44 Pg. 423-428. 1972.

HANSSON, A; VELIZ, G; NAQUIRA, C; AMREN, M; ARROYO, M; AREVALO, G. Preclinical and clinical studies with latex from Ficus glabrata HBK, a traditional intestinal antihelmintic in the Amazonian area. Journal of Ethnopharmacology. Vol. 17 (2). Pg. 105-138. 1986.

HERNANDEZ-MORALES, F; ASENJO, C.P. Inactivity of fresh pineapple juice as an anthelmintic in vivo. Journal Public Health Tropical Medicine. Porto Rico. 18 pp. 119. 1943.

JAFFE, W. The sap fig trees (as an Antihelmintic). Tropical diseases Bull. Venezuela. Vol. 40. Pg. 612. 1943.

KATO, S; BOWMAN, D.D; BROWN, D.L. Efficacy of Chenopodium ambrosioides as an antihelmintic for treatment of gastrointestinal nematodes in lambs. Journal Herbs Spices Medicinal Plants. Vol. 7(2) Pg. 11-25. 2000.

$\mathrm{KIM}$, N.D. Antihelmintic in crude drugs on the drugs for tapeworms. Yakhak Hoe Chi. Vol.19 Pg. 87. 1974.

KLIKS, MM. Studies on the traditional herbal antihelmintic Chenopodium ambrosioides Linn.: ethnopharmacological evaluation and clinical field trials. Tropical Society Medical. Pg. 879-887. 1985.

LUTTERMOSER, GW; BOND, HW. Antihelmintic activity of the fruits of Diospyros sp. and tests for activity of other persimmons. Helminthology Society. Vol. 24 Pg. 121. 1957.

MACIEL, M.V.; MORAIS, S.M.; BEVILAQUA, C.M.L.; CAMURÇA-VASCONCELOS, A.L.F.; COSTA, C.T.C. ; CASTRO, C.M.S. Ovicidal and larvicidal activity of Melia azedarach extracts on Haemonchus contortus. Veterinary Parasitology. Vol. 140. Pg. 98-104. 2006. MELLO, D. A.; PRIPAS, S.; FUCCI, M.; SANTORO, M. C.; PEDRAZZANI, E. S. Helmintoses intestinais Conhecimentos, atitudes e percepção da população. Revista de Saúde Pública. Vol. 22. Nº. 2. São Paulo. 1988.

Ministério da Saúde. Política nacional de plantas medicinais e fitoterápicos Departamento de Assistência Farmacêutica. - Brasília.. 60 p. Brasil. 2006.

NEVES, D. P. Parasitologia Humana. $11^{\text {a }}$ Ed. Editora. São Paulo. Atheneu. 2010. 494 p.

Organización Mundial de la Salud (OMS) (2002) Estrategia de la OMS sobre medicina tradicional.. Genebra: OMS. 2002-2005.

PEÑA, N; AURO, A; SUMANO, H. A comparative trial of garlic, its extract and ammonium-potassium tartrate as anthelmintics incarp. Journal of Ethnopharmacology. Mexico City. Mexico. Vol. 24 (2/3) Pg. 199-203. 1988.

PESSOA, L.M et al. Antihelmintic activity of essential oil of Ocimum gratissimum Linn. and eugenol against Haemonchus contortus. Veterinary Parasitology. Vol. 109. Pg. 59-63. 2002.

PHILLIPS, O. Ficus insipida (Moraceae): ethnobotany and ecology of an Amazonian antihelmintic. Economy Botany. Vol. 44 (4) Pg. 534-536. 1990.

RATES, S.M.K. Plants as source of drugs. Toxiconomy. Vol. 39. Pg. 603-13. 2001.

REIS, M.C.P.; et al. Experiencia na implantação do programa de fitoterapia do município do Rio de Janeiro. Revista Divulgação em Saúde para Debate. Pg. 4249. 2004.

RIOS-DE ÁLVAREZ, L., et al., In vitro screening of plant lectins and tropical plant extracts for antihelmintic properties. Veterinary Parasitology, doi:10.1016. 2011.

SANGSTER, N. C. Managing parasiticide resistance. Veterinary Parasitology. v. 98. p.89-109. 2001.

SANTOS, E. A. et al. Bioactivity evaluation of plant extracts used in indigenous medicine against the snail, Biomphalaria glabrata, and the larvae of Aedes aegypti. Evidence Based Complementary and Alternative Medicine. Volume 2012. 2012.

SCHENONE, H.; ROJAS, A.; GALDANES, M.; VILLAROEL, F.; HERNANDEZ, E. G.; CUEVA, R.; ORFALI, A.; PRIETRO, R.; ANGULO, M.; ORTEGO, M. Panorama de las helmintiasis intestinales humanas transmitidas por el suelo en Chile (1970-1980). Boletim Chileno del Parasitologia. Vol. 36. Pg.9-13. 1981.

SCHULZ et al.; Fitoterapia Racional. 4ª Ed. 2002.

SRINIVASA, B. et al. Antihelmintic activity of methanol extract of Amaranthus caudatus Linn. Journal of Food Safety. Vol.12. Pg.127-129. 2010.

TORRES, R. P.; SOUZA, M.A.F. A Dinâmica do Mercado Farmacêutico Brasileiro Segundo o Modelo das Estratégias Genéricas de Porter. Sociedade, Contabilidade e Gestão. Rio de Janeiro. Vol. 5, n. Especial. 2010.

VERA-MONTENEGRO, Y. et al. In vitro fasciolicide activity of some plant extracts against newly excysted flukes. Animal Biodiversity and Emerging Diseases: Annuary New York Academy of Sciences. Vol. 1149. 180-182. 2008.

VICHNEWSKI, W; GOULART, EG; HERZ, W. A heliangolide from Calea lantanoides Gard. Phytochemistry. Vol. 21 Pg. 464-465. 1982.

VINHA, C. Necessidade de uma política sanitária nacional para o combate às parasitoses intestinais. Revista da Sociedade Brasileira de Medicina Tropical.Vol. 10. Pg. 297-301. 1975.

WERNER, J. The sap of fig trees (as an anthelmintic). Tropical Diseases Bulletin. Vol. 40. Pg. 612. 1943.

WORKU, M; FRANCO. R; MILLER, J. H. Evaluation of the activity of plant extracts in Boer goats. American Journal of Animal and Veterinary Sciences. Vol. 4. No. 4. p. 72-79. 2009.

ZAMORA, JM; MORA, EC. Cytotoxic, antimicrobial and phytochemical properties of Larrea tridentata Journal of Ethnopharmacology. Vol. 45 (12) Pg. 3809-3810. 1985.

ZUANAZZI, J.A.S.; MAYORGA, P. Fitoprodutos e desenvolvimento econômico. Química Nova. Vol. 33. No. 6. Pag. 1421-1428. 2010.

Rev. Bras. PI. Med., Campinas, v.15, n.2, p.287-292, 2013. 